\title{
Spontaneous intralesional haemorrhage in dysembryoplastic neuroepithelial tumours: a series of five cases
}

\author{
Maria Thom, Beatriz Gomez-Anson, Tamas Revesz, William Harkness, Ciaran J O'Brien, \\ Rupert Kett-White, E Wyn Jones, John Stevens, Francesco Scaravilli
}

\begin{abstract}
Five patients with dysembryoplastic neuroepithelial tumour (DNT) showing extensive secondary haemorrhage, a finding not previously associated with these neoplasms, are described. The clinical presentations, neuroimaging findings, and histopathological features of these patients are reviewed. One patient, a previously asymptomatic 12 year old girl, presented with an acute intracerebral haemorrhage into a DNT. A further four young adults with histories of intractable partial and generalised seizures dating from childhood showed significant chronic haemorrhages within DNT, the MRI appearances in one patient giving a false impression of a cavernoma. Histopathology disclosed vascular abnormalities within these tumours which, together with other factors discussed, may have predisposed these tumours to haemorrhage.

(f Neurol Neurosurg Psychiatry 1999;67:97-101)
\end{abstract}

Keywords: DNT; epilepsy; hemorrhage; MRI

Dysembryoplastic neuroepithelial tumour (DNT) is a low grade, mixed neuronal and glial tumour usually associated with medically intractable complex partial or generalised seizures which date from childhood. The prognosis of these neoplasms is usually excellent after their identification with MRI and subsequent surgical excision ${ }^{12}$ and there has been only one report of malignant transformation. ${ }^{3}$

It is well recognised that an acute intralesional haemorrhage may mark the initial presentation of a primary intracranial glial tumour of both high and low grade ${ }^{4}$ and it has been suggested to be a particular liability in oligodendrogliomas, related to the prominent arcuate vascular patterns of these tumours. ${ }^{5}$ Vascular patterns similar to oligodendrogliomas are often present in $\mathrm{DNT}^{12}$ but there has previously been only one report documenting an asymptomatic haemorrhage within a DNT. ${ }^{6}$ We present five further DNTs with spontaneous haemorrhage, which complicated either the clinical presentations or the pathological diagnoses.

\section{Methods}

The five cases of DNT presented were encountered in the neurosurgical material examined at the National Hospital over a 2 year period (1994-6); histological sections from patient 1 were initially referred from the Morriston Hospital, Swansea, for review by one of us (TR). A review of all other DNTs on the hospital surgical pathology data base (28 tumours) found no similar cases. The clinical data from all patients were reviewed including seizure history, neuroimaging features, operative procedures, and follow up. The resected tissue was formalin fixed, paraffin embedded, and reviewed using conventional stains, including Perl's techniques for iron and immunohistochemistry (GFAP 1:400 Dako, and synaptophysin 1:20 Dako) for confirmation of the glial and neuronal components of the tumour. In three of the cases, intraoperative cytological smear preparations stained with toluidine blue were also available for review (patients 2, 4, and 5).

CLINICAL AND NEURORADIOLOGICAL FINDINGS AND SURGICAL TREATMENTS

Case 1

A previously well 12 year old girl presented with a short history of headache and confusion. Brain CT performed on admission showed a large acute intracerebral haemorrhage in the right temporoparietal region and angiography was negative. Six months later an MRI was performed for persisting headache which disclosed a well defined cystic mass in the right temporoparietal region (figure A) predominantly intracortical and involving the hippocampus and amygdala. It appeared hypointense on T1 and hyperintense on proton density and T2 weighted images and a small focus of residual subacute haemorrhage was seen in the posterior portion of the mass in the peritrigonal region (figure B). Minor central irregular enhancement was seen within the tumour after contrast administration. The patient underwent a right temporal lobectomy, with partial resection of the lesion after which she in revised form

15 October 1998

Accepted 29 October 1998 

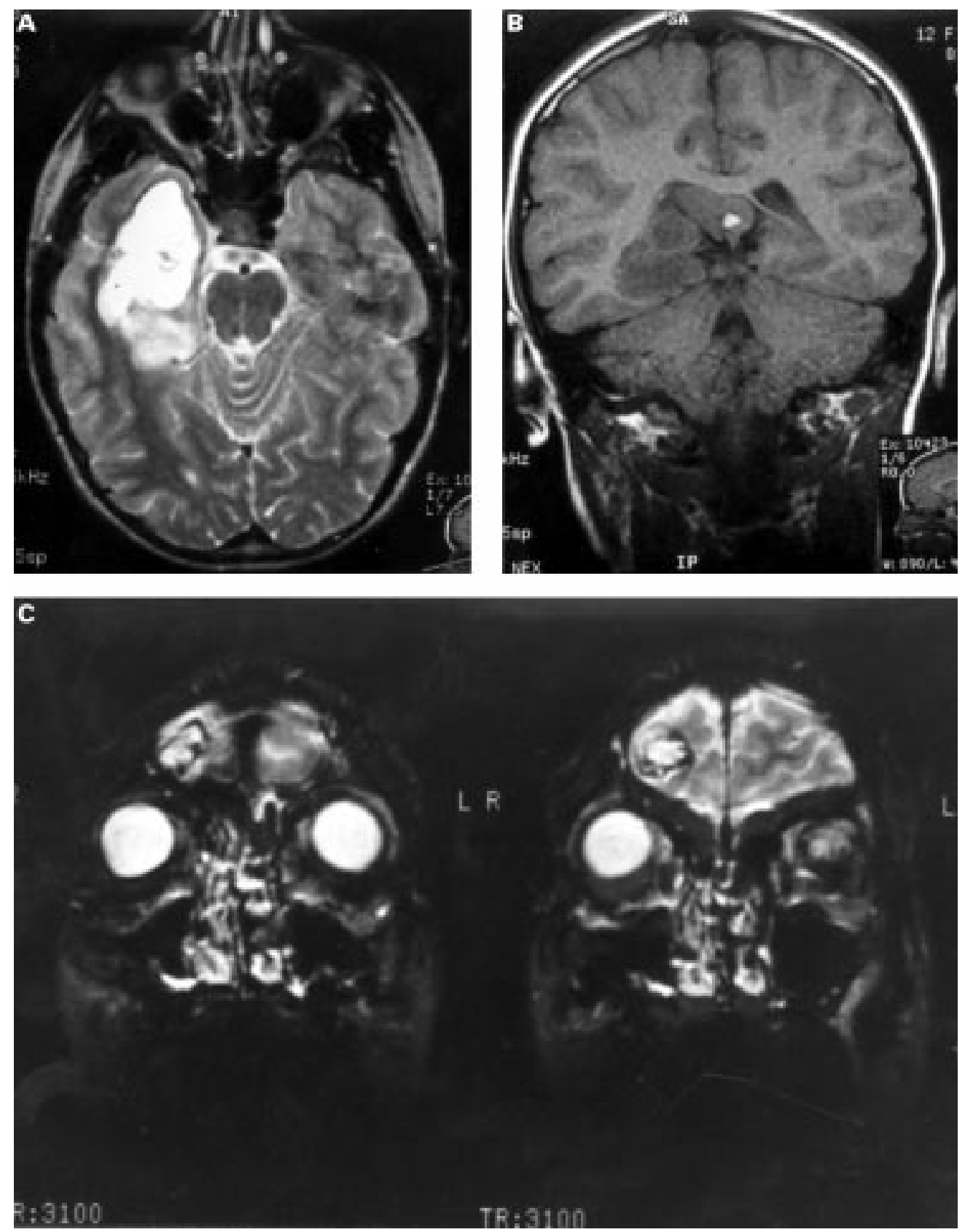

(A) (Case 1) T2 weighted axial MRI. Well demarcated, hyperintense mass in the right temporoparietal region. (B) (Case 1) T1 weighted coronal MRI. A small focus of subacute haemorrhage within the DNT below the splenium of the corpus callosum. (C) (Case 2) T2 weighted coronal MRI. A heterogenous, superficial right frontal DNT with a predominantly hyperintense centre and a hypointense rim representing haemosiderin from previous haemorrhage and giving a false impression of a cavernoma. (D) (Case 3) Specific glial-neuronal element which characterises DNT showing "floating" neurons and interspersed glial cells (bar=40 $)$. (E) (Case 1) A large, partly collapsed cyst within the DNT which abutts the leptomeninges. (Luxol fast blue. Bar=0.5 cm). (F) (Case 2) Dense, granular haemosiderin pigment deposition was present within the DNT impregnating the glial cells and scattered mature neuronal cells (haemotoxylin and eosin. Bar=50 $\mu$ ). $(G)$ (Case 1) Photomicrograph of mineralised blood vessels within DNT (haemotoxylin and eosin. Bar=24 $\mu$ ).

developed a left homonymous superior quadrantanopia but otherwise made a full recovery, is well at 1 year follow up, and has not at any time had seizures.

\section{Cases 2-5}

The clinical and operative details of these cases are summarised in the table. All patients had medically refractive seizures dating from childhood but none had histories of acute neurological deterioration to suggest episodes of intratumorous haemorrhage. The MRI appearances showed well demarcated, nodular, and cystic intracortical lesions involving temporal or frontal lobes. The signal characteristics in patients 3, 4, and 5 were typical of a DNT with lesional hypointensity on $\mathrm{T} 1$ and hyperintensity on proton density and T2 weighted images. However, in patient 2 the MRI was not typical, showing central CSF-like signal intensity, a peripheral rim of hypointensity on proton density, and T2 weighted images suggesting previous haemorrhage (figure $\mathrm{C}$ ) and surrounding hyperintensity in keeping with gliosis. These 

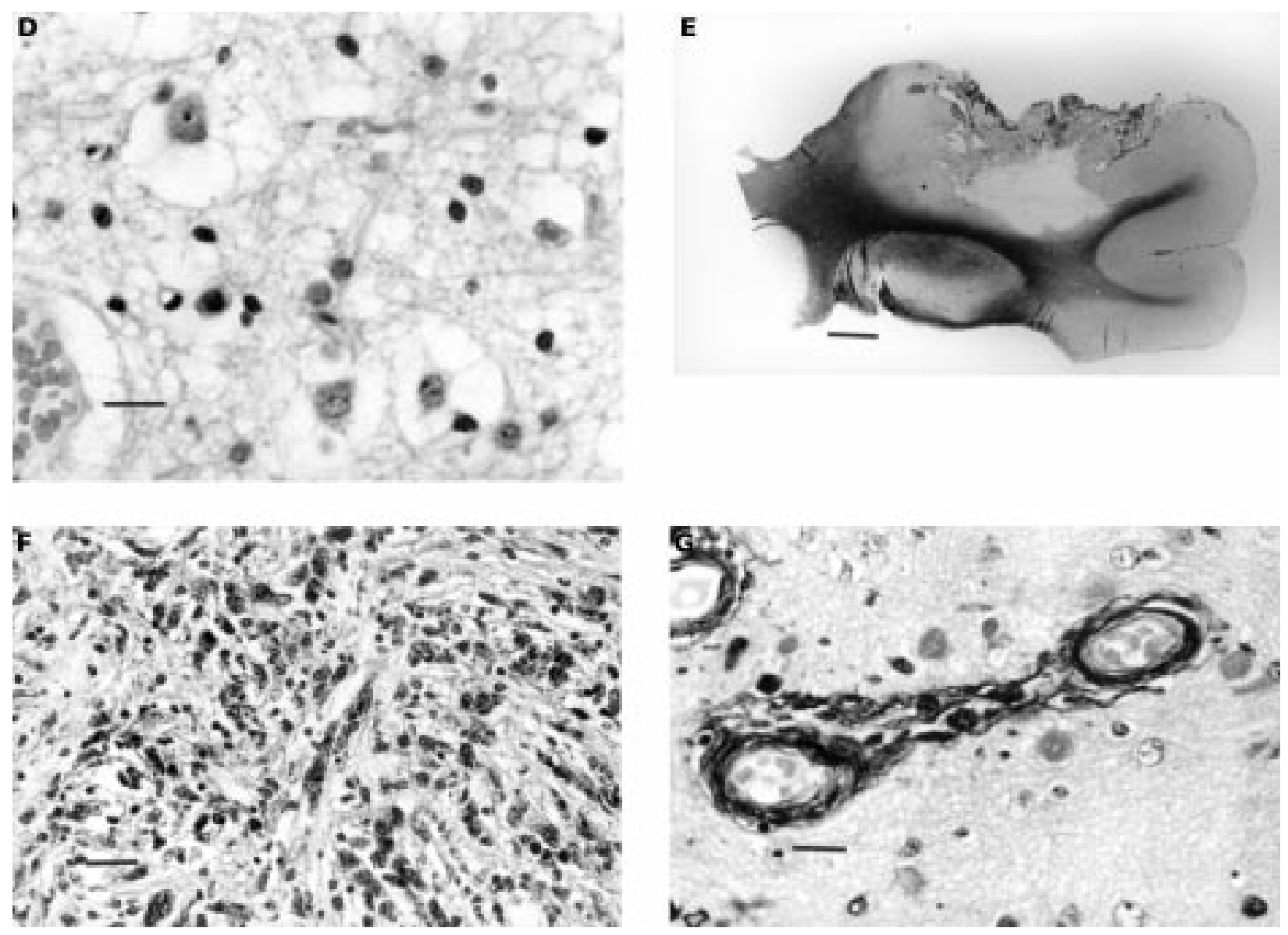

features led to a preoperative radiological diagnosis of a cavernoma. Preoperative serial MRI in patient 5 confirmed progressive enlargement of the tumour with cystic change between 1991 to 1995 . There was no radiological evidence of calcification either on CT or with MR in any of these tumours and in patients 2 and 3 contrast administration showed no enhancement and angiograms were negative.

HISTOLOGICAL FINDINGS

In two of three tumours in which cytological smear preparations were available for review, proliferations of astrocytes containing abundant haemosiderin pigment were present suggesting a cellular reaction to an old haemorrhage (patients 2 and 4 ); the third (patient 5) showed features of a low grade glioma consistent with a DNT. Surgical resections included two total (patients 2 and 3 ) and three partial

(patients 1, 4, and 5) lesionectomy specimens, and histological examination of these confirmed intracortical neuronal-glial tumours with a complex amalgam of architectural patterns as described in DNT. In some areas the normal cortex was replaced by tissue of a loosely structured mucinous nature, in which randomly distributed mature appearing nerve cells were "suspended" (figure D) and smaller oligodendroglial-like cells were clustered around thin walled capillaries representing the specific glioneuronal element of DNT. Well defined tumour nodules composed of rounded oligodendroglial-like cells and intermingled mature nerve cells were seen and also areas with pilocytic astrocytoma-like growth pattern were present in two (patients 1 and 2). All patients showed extension of tumour into the leptomeninges. Formation of cystic cavities of varying size were present within all the

Clinical histories and operative details of patients 2 to 5

\begin{tabular}{|c|c|c|c|c|c|}
\hline Patient Sex & $\begin{array}{l}\text { Age at } \\
\text { onset of } \\
\text { seizures } \\
\text { (y) }\end{array}$ & $\begin{array}{l}\text { Seizure type and approximate frequency } \\
\text { before surgery }\end{array}$ & $\begin{array}{l}\text { Age at } \\
\text { surgery } \\
\text { (y) }\end{array}$ & Tumour location and surgical procedure & Outcome and duration of follow up \\
\hline 2 Male & 7 & $\begin{array}{l}\text { Partial (1/week) and generalised seizures } \\
(1 / \text { week }) \text {. }\end{array}$ & 24 & $\begin{array}{l}\text { Right frontal. Removal of cystic mass } \\
\text { (total excision). }\end{array}$ & $\begin{array}{l}\text { Only one seizure since surgery. } \\
\text { Postoperative EEG shows no } \\
\text { epileptiform activity. } 2 \text { year follow up }\end{array}$ \\
\hline 3 Male & 9 & $\begin{array}{l}\text { Complex partial seizures and } \\
\text { generalized seizures (2-3/week). }\end{array}$ & 27 & $\begin{array}{l}\text { Left temporal. Lesionectomy; lower } \\
\text { middle and inferior temporal gyrus. }\end{array}$ & Seizure free. 18 month follow up. \\
\hline 4 Female & 6 & $\begin{array}{l}\text { Absence and generalised seizures } \\
\text { ( } 2 / \text { week). }\end{array}$ & 20 & $\begin{array}{l}\text { Left frontal. Subtotal ( } 80 \%) \text { excision: } \\
\text { due to tumour location in motor cortex. }\end{array}$ & $\begin{array}{l}\text { Continues to have seizures, although } \\
\text { decreased in frequency ( } 2 \text { month). } 18 \\
\text { month follow up. }\end{array}$ \\
\hline 5 Female & 6 & $\begin{array}{l}\text { Complex partial seizures ( } 5-10 / \text { month) } \\
\text { and generalised seizures ( } 5 / \text { year). }\end{array}$ & 41 & $\begin{array}{l}\text { Right frontal. Cystic lesion extending to } \\
\text { cortex. Cyst drained and partial } \\
\text { resection of tumour. }\end{array}$ & $\begin{array}{l}\text { Marked reduction in seizure frequency. } \\
1 \text { year follow up. }\end{array}$ \\
\hline
\end{tabular}


tumours, many close to the meningeal surface (figure E). Adjacent cortical dysplasia was noted in three patients $(1,2$, and 3$)$

Extensive intratumorous haemosiderin deposition was confirmed with Perl's staining; the iron pigment was abundant in cyst walls and also diffusely impregnating tumour cells (figure F). The tumour vasculature was composed predominantly of delicate thin walled branching capillaries, typical of the arcuate pattern previously described in DNT, and was present in both tumour nodules and the glioneuronal elements of all cases. These vessels traversed through the mucinous matrix of the tumour where they appeared relatively unsupported. In two (patients 1 and 5) glomeruloid tufts of proliferating capillaries were present in close proximity to areas of haemorrhage. Extensive mineralisation of vessels (figure $G$ ) was found in two tumours (patients 1 and 3 ). In none of the cases were vascular endothelial proliferations characteristic of malignant astrocytoma seen and there was no evidence of vascular thrombosis, amyloid deposition, or angiitis.

\section{Discussion}

Spontaneous intratumorous haemorrhage may mark the initial presentation of both low and high grade glial neoplasms. ${ }^{45}$ DNTs are distinctive low grade glial-neuronal neoplasms typically associated with intractable epilepsy dating from childhood. There is a single, previous report of a DNT in which a chronic haemorrhage was found ${ }^{6}$ but an acute presentation with spontaneous intratumorous haemorrhage in a histologically established DNT has not been previously documented. In a further four DNTs we have histological evidence of chronic haemorrhage which in the absence of episodes of acute neurological deterioration, we can suspect has accumulated over time from small clinically silent bleeds. In three of the four patients, the haemorrhage was not visualised by MRI, which may indicate smaller amounts of haemosiderin deposition in these patients.

None of the patients had evidence of a coagulopathy and we examined the tumour vasculature as a potential factor which may have predisposed these tumours to haemorrhage. The angiograms, when performed, were normal and enhancement with contrast MRI was only seen focally in patient 1,6 months after an acute cerebral haemorrhage. Although this enhancement may have been interpreted as neovascularisation of an organising haematoma within the tumour, it was considered more likely to represent abnormal vessels within the tumour itself. Enhancement on MRI within DNTs is not in itself an unusual finding and has been reported in up to $36 \%$ of patients in one series and in six of 16 patients in another. ${ }^{26}$

Histologically, the predominant vascular patterns in the neoplasms were fine delicate, branching capillary networks, also termed arcuate or retiform vessels. Similar vascular patterns have been shown to be significantly associated with tumour haemorrhage in a study of gliomas. ${ }^{5}$ It was also noted in this same study that haemorrhage within oligodendrogliomas correlated with calcification of such tumour vessels. We also found dystrophic calcification or mineralisation of small vessels in two of the present cases. It is not inconceivable that such calcified vessels may have impaired haemostatic vasoconstrictive reponses and so contribute to the extent of haemorrhage. Additionally, we noted that the fine capillaries within the DNT characteristically traverse through the abundant mucoid matrix of the tumour, relatively unsupported by astrocytic processes. Such vessels may be more vulnerable to mechanical shearing during episodes of minor cerebral trauma, a not uncommon occurrence in patients with repeated and poorly controlled generalised seizures. Also present in two patients were glomeruloid-like tufts of proliferating capillaries in close proximity to areas of haemorrhage suggesting a causal link, although in a previous study of gliomas no significant association between the presence of haemorrhage and glomeruloid vascular pattern was found. ${ }^{5}$ These vascular abnormalities have occasionally been noted in previous published series of DNTs; ${ }^{12}$ however, in the only previously documented case of a DNT with haemorrhage, no mention of abnormal vasculature was recorded. ${ }^{6}$

Cystic degeneration, a not infrequent finding in DNT, ${ }^{67}$ was present in all the cases on MRI and confirmed histologically, many of the cysts containing old blood pigment suggesting they may have been a factor contributing to the accumulative size of the haemorrhages. Also, invasion of the leptomeninges by these superficial tumours was common to all, with large cysts closely opposed to the frontal or temporal cortical surface (figure $\mathrm{C}$ ) which may also have increased their vulnerability to traumatic haemorrhage.

We can hypothesise that the iron deposited within these tumours played a functional role in seizure propagation and worsening of the epilepsy in these cases. Iron is known to precipitate epilepsy both in clinical and experimental situations; free radical formation and lipid peroxidation of neural membranes ${ }^{8}$ and excitatory neurotransmitter release $^{9}$ are possible mechanisms for seizuregenesis in this model of epilepsy. The role of iron in enhancing epileptiform activity within these DNTs, however, remains highly speculative, particularly as in patient 1 there was no history of seizures either before or after partial tumour resection.

The are several diagnostic considerations arising from this group of patients: firstly, that DNT may rarely present with an acute intracerebral haemorrhage as in the first patient of this series. This case also highlights the potential value of $M R$ neuroimaging in young patients with angiogram-negative intracranial haemorrhage as has been noted in a previous study. ${ }^{10}$ It was also remarkable in the absence of a history of seizures which although unusual for DNT, has occasionally been seen. ${ }^{2}$ We have also shown that clinically silent, chronic haemorrhages may accumulate in DNT, and significantly alter the MRI appearance giving a false impression of a cavernoma. Lastly, the interpretation of the 
intraoperative cytological smear preparations may be complicated by the old haemorrhage and reactive gliosis present in such DNTs. We therefore conclude that the presence of haemosiderin pigment on smears which otherwise show appearances typical of a DNT should not deter the pathologist from making this diagnosis and thus reassure the surgeon to proceed with the appropriate lesionectomy.

B G-A was funded by the Spanish ministry of health (FISS $96 / 5570$ and $97 / 5158)$. A preliminary publication of this work has appeared in abstract form Neuropathol Appl Neurobiol 1997;23:443 and was presented at the 94th meeting of the British Neuropathological Society.

1 Daumas-Duport C, Scheithauer BW, Chodkiewicz JP, et al. Dysembryoplastic neuroepithelial tumour: a surgically curable tumour of young patients with intractable partia seizures. Report of 39 cases. Neurosurgery 1988;23:545-56.

2 Raymond AA, Halpin SFS, Alsanjari N, et al. Dysembryoplastic neuroepithelial tumour: features in 16 patients. Brain 1994;171:461-75
3 Hammond RR, Ranger AM, Woulfe JMJ, et al. The malignant potential of dysembryoplastic neuroepithelial tumours (DNETs) [abstract]. F Neuropathol Exp Neurol 1997;56:606.

4 Barth H, Fritsch G, Haaks. Intracerebral hematoma as an acute manifestation of intracranial tumours. Nervenarzt 1994;65:854-8.

5 Liwnicz BH, Wu SZ, Tew JM. The relationship between the capillary structure and haemorrhage in gliomas. $\mathcal{F}$ Neurosurgery 1987;66:536-41.

6 Ostertun B, Wolf HK, Campos MG, et al. Dysembryoplastic neuroepithelial tumours: MR and CT evaluation. Am $\mathcal{f}$ Neuroradiol 1996;17:419-30.

7 Prayson RA, Morris HH, Estes ML, et al. Dysembryoplastic neuroepithelial tumour: a clinicopathologic and immunohistochemical study of 11 tumours including MIB1 immunoreactivity. Clin Neuropathol 1996;15:47-53.

8 Yokoi I, Toma J, Liu J, et al. Adenosines scavenged hydroxyl radicals and prevented post traumatic epilepsy. Free Radic Biol Med 1995;19:473-9.

9 Janjua NA, Mori A, Hiramatsu M. Increased aspartic acid release from the iron induced epileptogenic focus. Epilepsy Res 1990;6:215-20.

10 Renowden SA, Molyneux AJ, Anslow P, et al. The value of MRI in angiogram-negative intracranial haemorrhage: Neuroradiology 1994;36:422-5. 